\title{
Estructura factorial del inventario multicultural de la depresión, estado-rasgo: Rol de las emociones positivas en la depresión
}

\author{
Manolete S. Moscoso, Cecile A. Lengacher \& Melissa Knapp \\ University of South Florida
}

Recibido: 29 de abril del 2012 / Aceptado: 1 de junio del 2012

El propósito de este artículo fue presentar la construcción del inventario multicultural de la depresión, estado-rasgo (Imuder).Tomando en consideración el marco teórico de Susan Folkman acerca de la coexistencia de emociones positivas y negativas en el proceso de estrés severo y depresión, se procedió a la elaboración de items caracterizando estados y rasgos de emociones que expresan la presencia o ausencia de depresión. En base a una muestra multicultural con participantes de diversos países latinoamericanos, se realizó el análisis factorial de componentes principales con rotaciones promax, a fin de determinar la validez de constructo y la consistencia interna del instrumento.

depresión / personalidad / estrés / validez de constructo / investigación transcultural

Factorial structure of the multicultural state-trait depression inventory: The role of positive emotions in depression

The principal purpose of this study was to report the development of the multicultural state-trait depression inventory (Mustdi). We present the theoretical framework developed by Susan Folkman about the co-existence of positive and negative emotions during the experience of severe stress and depression. We developed a set of items as emotional states and traits that express the presence or absence of depression. Responses to the items, were factored in separate principal factor analysis with promax rotations for state and trait scales to determine the construct validity and internal consistency of the instrument.

depression / personality / chronic stress / cross-cultural research / construct validity

Correos electrónicos: mmoscoso@health.usf.edu; clengach@health.usf.edu 
La Organización Mundial de la Salud (OMS) calcula que 340 millones de personas sufren actualmente de alguna de las formas de depresión clínica. Asimismo, la OMS indica que la depresión llegará a ser la primera causa de discapacidad y la segunda causa, después de la obesidad, que contribuya a la adquisición de enfermedades en el mundo para el año 2020 (WHO, 2008). Se estima que en Estados Unidos los desórdenes depresivos afectan aproximadamente al $17 \%$ de la población en algún momento de sus vidas (Kessler et al., 2005). Debido a este elevado nivel de prevalencia, no sorprende que la depresión sea descrita como "el resfriado de la salud mental, el cual afecta a ricos y pobres, mujeres y varones, así como también jóvenes y adultos" (Rosenfeld, 1999, p. 10). La Asociación Psiquiátrica Americana reporta que por lo menos el $60 \%$ de individuos que experimentaron un primer episodio de depresión, volverán a experimentar un segundo episodio; un $70 \%$ de personas que experimentan un segundo episodio, experimentarán una recaída por tercera vez; y el $90 \%$ de pacientes con 3 episodios volverán a experimentar una cuarta crisis (APA, 2000a).

Estas cifras indican claramente que una proporción significativa de personas en el mundo son afectados por esta enfermedad. Aproximadamente un 20\% de estos pacientes no responden adecuadamente a los tratamientos disponibles y sufren la recurrencia del trastorno (Fava,
2003). Una de las grandes limitaciones en el estudio de la depresión es su evaluación y diagnóstico. El hecho de que este desorden es actualmente definido por el DSM-IV (APA, 2000a) como "simplemente un conjunto de criterios o síntomas" que se presentan "en un periodo de tiempo" ha sido ampliamente criticado en los ámbitos académico y clínico (Beck, et al., 2011). Nos urge dejar de lado la idea de que la depresión es simplemente una constelación de síntomas aislados. De igual manera, es importante entender que la depresión no es únicamente un problema clínico y epidemiológico en salud mental, sino un problema de salud en medicina.

La evaluación psicométrica de la depresión necesita adecuarse a las nuevas pautas de conceptualización que toman en consideración las emociones positivas y estilos de afrontamiento positivo dentro del proceso de estrés (Folkman, 1997, 2008). Este nuevo enfoque sugiere incluir formas más dinámicas de evaluación como los rasgos de personalidad y sus diferencias individuales, de manera que podamos concebir a la depresión como un desorden más coherente encaminado hacia un mejor entendimiento de este trastorno (Bolger \& Zuckerman, 1995). Es pertinente indicar que el Instituto de Salud Mental de Estados Unidos ha formado un comité de revisión con el propósito de evaluar las clasificaciones diagnósticas de los desórdenes mentales, incluyendo la depresión (Insel et al., 2010). 
En referencia a la medición psicométrica de la depresión, Beck, Steer \& Brown (1996) hacen mención del carácter confuso de ítems representativos de síntomas somáticos en instrumentos de evaluación de la depresión, especialmente cuando se utilizan estas escalas en pacientes que sufren enfermedades físicas. Es importante indicar que en el caso de pacientes deprimidos con diagnóstico de enfermedades físicas, los "síntomas somáticos" están directamente relacionados con los efectos secundarios de sus tratamientos como sucede en los casos del cáncer, la artritis, las enfermedades cardiovasculares, la fibromialgia, la fatiga crónica y la osteoporosis. Por otro lado, ciertos instrumentos psicométricos basan su evaluación únicamente en la ausencia o presencia de síntomas de la depresión, así como en el nivel de severidad. Entre estas pruebas tenemos la escala de Hamilton, la cual tiende a elevar el riesgo de infravaloración del trastorno depresivo (Mallon y Hetta, 2002).

La claridad conceptual es esencial para el progreso científico. En este sentido, la elaboración de un instrumento psicométrico válido y confiable para la evaluación de la depresión necesita distinguir las características de personalidad y diferencias individuales con los cuales esta enfermedad está relacionada de manera directa o indirecta. Reitterban y Spielberger (1996) reportaron un primer avance y pusieron en evidencia las deficiencias de los actuales métodos de evaluación psicométrica de la depresión observando la tendencia de algunos instrumentos a evaluar la intensidad del trastorno depresivo utilizando instrucciones que indican más bien la frecuencia de los síntomas. En un segundo estudio, Spielberger, CarreteroDios, De los Santos-Roig y Buela-Casal (2002) refieren que dicho problema confunde las puntuaciones entre intensidad (estado) y frecuencia (rasgo), presentando una alternativa psicométrica para remediar estas deficiencias: The Spanish Experimental version of the State-Trait Depression Questionnaire, cuyo objetivo principal fue identificar el grado de afectación del componente afectivo de la depresión. A pesar de ser un avance innovador en la medición psicométrica de la depresión, consideramos que esta nueva forma de evaluación es aún incompleta, en virtud de mantener la conceptualización de la depresión como un proceso estático, es decir entendido como una "constelación de síntomas" basados en los criterios de distimia indicados en el DSM-IV.

Los argumentos presentados en los párrafos anteriores nos permiten concluir, en primer lugar, que los instrumentos actuales de medición de la depresión ofrecen categorías diagnósticas ambiguas y no diferencian de una manera objetiva las dimensiones de los trastornos depresivos. En segundo lugar, las escalas de medición disponibles han sido elaboradas con el propósito de evaluar la intensidad de los sintomas de 
la depresión indicando la frecuencia de ocurrencia de estos en base únicamente a las definiciones presentadas por el DSM-IV. En tercer lugar, las pruebas psicométricas actuales carecen de un modelo conceptual que describa la depresión como una entidad coherente y dinámica, es decir, como un cuadro psicopatológico que se presenta dentro del proceso de afrontamiento de eventos estresantes de la vida y su interacción con las diferencias individuales de personalidad.

\section{EL PROCESO DE ESTRÉS PERCIBIDO Y DEPRESIÓN}

En la actualidad, tenemos una significativa cantidad de estudios empíricos que demuestran de manera concluyente la relación directa entre los eventos estresantes y la depresión. La pregunta que nos debemos plantear es: ¿cuáles son los mecanismos de afrontamiento y diferencias individuales por los que el estrés produce estados depresivos? Todos estamos expuestos a los diferentes eventos estresantes de la vida; sin embargo, no todos experimentamos trastornos depresivos. ¿Por qué? Para responder estas preguntas debemos tomar en consideración el concepto de estrés percibido como un tema central al proceso de estrés, afrontamiento y depresión. En este sentido, es importante mencionar que una de las características importantes dentro del proceso de estrés es el concepto de appraisal o evaluación cognitiva, el cual considera un elemento importante: La percepción del individuo en relación con el "estresor" (Lazarus \& Folkman, 1984).

El ingrediente principal de la respuesta emocional del estrés es de naturaleza cognitiva debido al hecho de que el "evento externo o estresor deberá ser percibido como estresante". Un significativo número de estudios empíricos encuentran una relación directa entre eventos estresantes y el inicio de episodios depresivos (Kendler \& Prescott, 1998). Resultados de estudios en esta área indican que los estresores son factores de riesgo para el desarrollo de trastornos depresivos, particularmente en individuos con un elevado nivel de emociones negativas y de vulnerabilidad (Mayberg, 2004). Estudios con muestras de poblaciones no clínicas reportan que hasta un $80 \%$ de individuos que experimentan una crisis de estrés, desarrollan episodios depresivos (Mazure, 1998). Estos mismos estudios indican que episodios depresivos causados por eventos estresantes agudos llevan un mejor pronóstico en comparación con episodios depresivos causados por el estrés crónico. Debemos indicar que el estrés crónico tiene un mayor impacto sobre la depresión que los eventos estresantes agudos, particularmente en relación con trastornos depresivos que tienen periodos largos de duración (Kendler \& Prescott, 1998).

La relación entre el estrés y los episodios depresivos se fundamenta en la afinidad del estilo de personalidad del 
individuo, en función de vulnerabilidad o resiliencia, y la forma de percepción de estresores cuyo contenido sea relevante a cierto estilo cognitivo y emocional de la personalidad. La literatura actual demuestra empíricamente que el balance de emociones positivas y emociones negativas permiten predecir el nivel de bienestar personal y de la salud del individuo (Diener, Sandvik \& Pavot, 1991). Larson, Nitschke \& Davidson (2007) indican que la capacidad de mantener un adecuado nivel de emociones positivas y reducir los niveles de emociones negativas se encuentran notoriamente alteradas en pacientes que experimentan desórdenes depresivos. De igual manera, los estudios de Folkman con pacientes de VIH demuestran que las emociones positivas y las emociones negativas coexisten y se presentan de manera simultánea durante los periodos de estrés severo y depresión. A manera de ilustración debemos incluir el comentario extraído literalmente del artículo de Folkman (1997) acerca de la pareja de un paciente de VIH fallecido días antes a la fecha de su entrevista, el cual nos permite entender este proceso de manera más clara: "Existe una dualidad en mi vida. Siento una gran tristeza causada por la muerte de Jim, sin embargo, siento que tengo, a la misma vez, la habilidad de gozar de la vida. Simplemente necesito aceptar el hecho de que ambos aspectos están en mí, y ambos coexisten".
MARCO CONCEPTUAL DEL INVENTARIO MULTICULTURAL DE LA DEPRESIÓN, ESTADORASGO

La elaboración y adaptación de ítems del inventario multicultural de la depresión, estado-rasgo (Imuder), está fundamentada en el modelo conceptual de Susan Folkman (1997, 2008). Este modelo incluye las emociones positivas y los estilos de afrontamiento positivo dentro de la interacción del proceso de estrés severo y depresión. Folkman y colaboradores sugieren tres estilos de afrontamiento por los cuales las personas con niveles elevados de distrés y depresión logran expresar emociones positivas:

1) Re-evaluación cognitiva positiva (Positive reappraisal), en la cual un individuo logra percibir el lado positivo de una experiencia estresante ("pienso que los fracasos son parte de la vida", "siento que soy una persona alegre", "siento que soy una persona sociable").

2) Afrontamiento orientado a metas (Goal-focused coping), en que la persona mantiene una postura orientada hacia actividades de la vida diaria y una perspectiva futura positiva ("me siento entusiasmado con mis proyectos", "me siento interesado en el futuro", "pienso que mi futuro es brillante").

3) Infusión de eventos ordinarios con un significado positivo (Positive meaning) por medio del cual el indi- 
viduo materializa sus actividades con un significado positivo ("me encan$\tan$ mis actividades laborales", "me siento feliz en mis relaciones sociales", "soy una persona llena de vitalidad", "siento que disfruto la vida").

El modelo conceptual de Folkman recibe un significativo apoyo de las investigaciones desarrolladas por Fredickson (1998), cuya labor empírica estimuló considerablemente el estudio de las emociones positivas. Fredickson indica que la experiencia de emociones positivas amplian el proceso cognitivo y de acción del individuo, extendiendo el enfoque atencional y generando un repertorio de conductas que sirven de base para construir recursos personales y de resiliencia para el afrontamiento del estrés (Fredickson, 2003). Dentro de

Figura 1

Modelo conceptual del inventario multicultural de la depresión, estado/rasgo

Estrés, personalidad y depresión

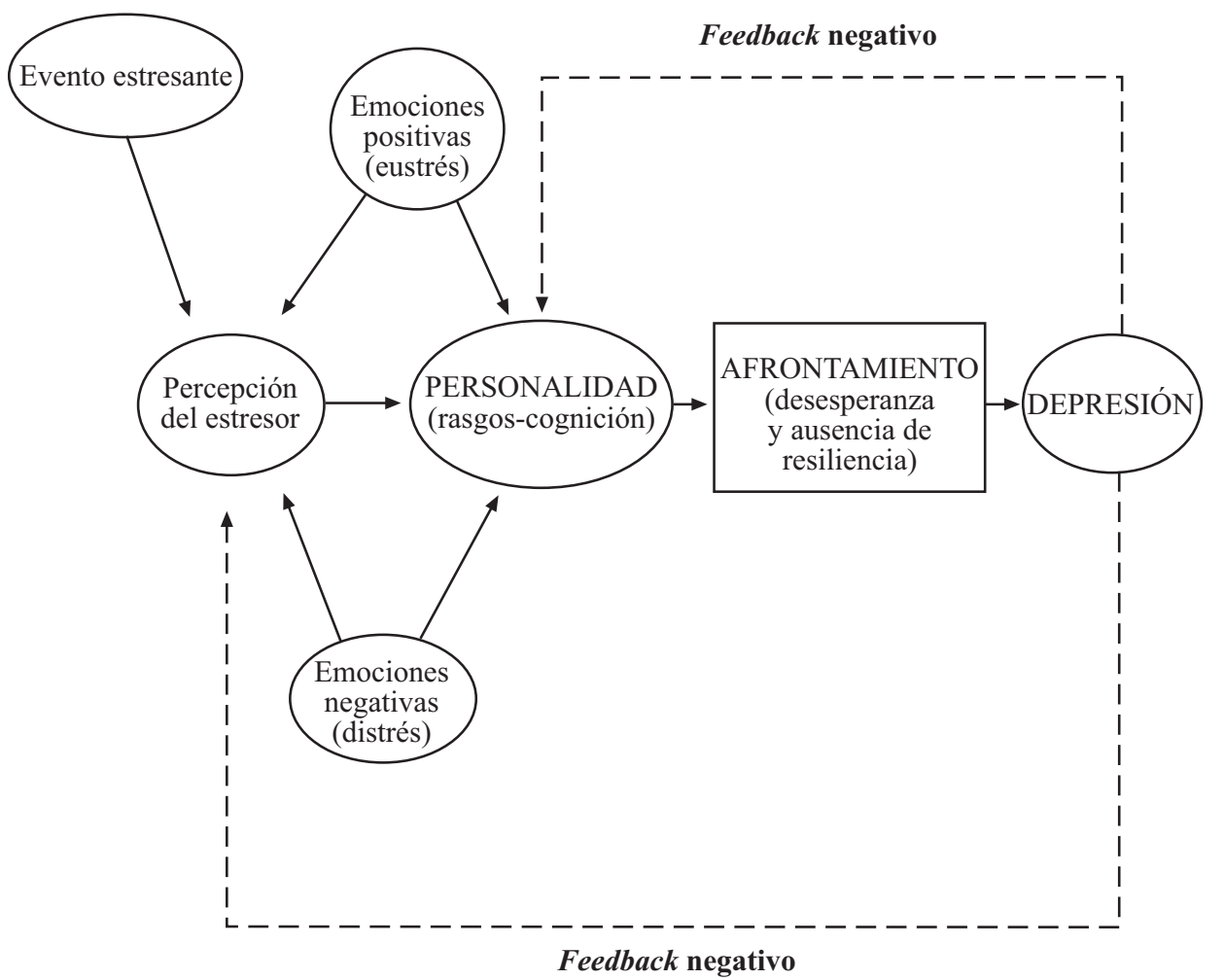


esta misma línea de investigación, las investigaciones de Seligman acerca de la psicología positiva continúan estimulando un buen número de estudios que reportan los beneficios de las emociones positivas en el afrontamiento del estrés, depresión, y de la salud en general (Seligman \& Csikszentmihalyi, 2000).

Los estudios desarrollados por Zautra (Zautra et al., 2005) permitieron una genuina expansión del trabajo de investigación de Folkman acerca de la existencia de las emociones positivas dentro del proceso de estrés. Zautra (2003) sugiere que las emociones positivas promueven la adaptación psicológica y fisiológica del individuo con significativos beneficios para la salud. Zautra y Smith (2001) refieren que las emociones positivas ofrecen información valiosa acerca de la vida de un individuo, las cuales pueden ser de gran utilidad para diferenciar los perfiles de percepción en el caso de un trastorno depresivo o de salud en general. Los estudios de Folkman, de la misma manera, reciben un soporte empírico muy significativo por parte de los estudios realizados por Clark y Watson (1991) en cuanto a la afectividad positiva y la afectividad negativa y su interacción con el proceso de estrés percibido. Estos autores observaron cambios y deficiencias significativas en la afectividad positiva únicamente en pacientes depresivos (Watson \& Clark, 1994). De igual manera, Kendall y Watson (1989) refieren que los ítems relacionados con depresión presentan cargas elevadas en la afectividad negativa.

Finalmente, en la elaboración del Imuder tomamos en consideración la distinción "estado-rasgo" planteada por Cattel y Scheier en 1961, la cual ha demostrado ser de gran utilidad en la evaluación de la ansiedad (Spielberger, Gorsuch \& Lushene, 1970) y la expresión de la ira (Spielberger, 1988). Esta distinción metodológica nos sirvió de marco referencial en nuestros estudios sobre la experiencia, expresión y control de la ira en Latinoamérica durante la década anterior (Moscoso \& Spielberger, 1999; Spielberger, Moscoso \& Brunner, 2005), y recientemente en estudios sobre la depresión en Europa y Estados Unidos (Spielberger, et al., 2002; Spielberger, Ritterband, Reheiser \& Brunner, 2003).

El propósito principal de nuestro estudio es presentar el modelo conceptual que sirvió de guía en la elaboración y adaptación de los ítems del inventario multicultural de la depresión, estadorasgo (Imuder), en su forma preliminar, y reportar su estructura factorial y consistencia interna en una muestra poblacional no clínica y multicultural de adultos de habla hispana.

\section{ELABORACIÓN DEL INVENTARIO} MULTICULTURAL DE LA DEPRESIÓN, ESTADORASGO

El inventario multicultural de la depresión, estado-rasgo (Imuder) es el resultado de nuestro trabajo de investi- 
gación psicométrica transcultural en la Universidad de South Florida, Estados Unidos. El propósito fue desarrollar un instrumento psicométricamente válido y confiable en el contexto de las interacciones de las emociones positivas dentro del proceso de estrés. El Imuder evalúa la depresión como una entidad coherente, multidimensional y dinámica en poblaciones no clínicas bajo condiciones de estrés agudo y crónico.

Los ítems del Imuder están construidos de manera que reflejen la presencia de la depresión como función de características cognitivas y emocionales (emociones negativas); y la ausencia de síntomas depresivos expresados en función de rasgos cognitivos y emociones positivas. Es importante indicar que este instrumento psicométrico no toma en consideración los "síntomas somáticos", como por ejemplo, pérdida de peso, insomnio, dolor, pérdida de apetito, comúnmente asociados con enfermedades físicas, con el propósito de evitar comprometer la validez y confiabilidad del constructo de depresión, al ser utilizado en pacientes que sufren enfermedades crónicas (Moscoso, McCreary, Goldenfarb, Knapp \& Rohr, 1999; Moscoso \& Reheiser, 2010).

En nuestro primer estudio acerca del Imuder se incluyó ítems sustentados en la validez de contenido y marco conceptual de la depresión descrito en líneas anteriores. Se tomó en consideración el grado de entendimiento y dificultad para responder a cada ítem en base a las instrucciones utilizadas para las escalas estado-rasgo. Se elaboró un grupo inicial de 36 ítems con el propósito de medir dimensiones emocionales que expresen la presencia o ausencia de depresión en el contexto del estrés percibido (Moscoso, Knapp \& Llauget, 2003).

Se utilizó la distinción estado-rasgo desarrollada por Cattel y Scheier para la construcción de dos escalas con el propósito de evaluar la depresión/estado y la depresión/rasgo. Se incluyó instrucciones propias para cada una de las escalas, como sucedió en la adaptación del inventario multicultural latinoamericano de la cólera y hostilidad (Moscoso, 2000). Algunos de los 36 ítems fueron adaptados de otras pruebas de medición, como el inventario de depresión de Beck (Beck \& Steer, 1993), el inventario de distrés emocional percibido (Moscoso \& Reheiser, 2010) y la escala de depresión del inventario de personalidad estado-rasgo de Spielberger (Spielberger et al., 1979; Spielberger et al., 2003).

El inventario preliminar de 36 ítems fue presentado a un grupo de 18 jueces conformado por profesionales de la salud y pacientes con diagnóstico de cáncer hispanoparlantes, como parte del plan piloto para evaluar la validez de contenido del instrumento, tomando en consideración el grado de dificultad para entender y responder cada ítem. Del grupo de ítems construidos inicialmente, 12 (33\%) fueron eliminados en 
virtud de las evaluaciones y comentarios de los jueces con relación al grado de dificultad y especificidad para entender el ítem, así como su singularidad dentro del grupo. Este proceso permitió la eliminación de los reactivos con contenido redundante o confuso en cuanto al concepto de depresión. Decidimos retener 24 ítems (12 ítems/estado y 12 ítems rasgo) con los cuales llevamos a cabo los análisis de confiabilidad y consistencia interna en una muestra de 172 pacientes hispanoparlantes con diagnóstico de cáncer en el Morton Plant Hospital de Clearwater, Florida. Dichos análisis se realizaron en base al coeficiente Alpha de Cronbach y las pruebas de correlación test-retest en un intervalo temporal de dos meses con el propósito de observar el grado de estabilidad del instrumento (Moscoso et al., 2003).

El Imuder, en su forma preliminar, es una prueba confiable, con una correlación Pearson test-retest de 0.43 en la escala de depresión/estado y 0.82 en la escala de depresión/rasgo. El valor relativamente bajo en la escala depresión/ estado se explica debido a la naturaleza temporal de los estados depresivos. Los resultados en base al coeficiente alpha de Cronbach indican una elevada consistencia interna de sus escalas, con valores de 0.87 para varones y 0.90 para mujeres en la escala de depresión/ estado; 0.89 en varones y 0.91 en mujeres para la escala de depresión/rasgo. Estos resultados preliminares nos indi- can claramente que dichos ítems son de naturaleza homogénea (Moscoso et al., 2003).

Teniendo como base los resultados iniciales encontrados en nuestro estudio piloto en Florida, Estados Unidos, decidimos continuar nuestra investigación instrumental en el marco del 30. Congreso Interamericano de Psicología realizado en Buenos Aires. El objetivo principal de esta segunda fase fue analizar la estructura factorial de componentes principales con rotaciones promax del Imuder, en su forma preliminar, y continuar evaluando la validez de constructo y consistencia interna en una muestra de población no clínica y multicultural con adultos de habla hispana. El presente artículo reporta los resultados de este segundo estudio.

\section{Método}

\section{Participantes}

La muestra utilizada para el estudio del análisis factorial y consistencia interna del Imuder está compuesta por un total de 526 participantes (324 mujeres y 202 varones) del 30. Congreso Interamericano de Psicología realizado en la ciudad de Buenos Aires, Argentina. Se obtuvo información demográfica de cada sujeto en relación con edad, nacionalidad, género y nivel de educación. Las edades de los sujetos varían entre 22 y 66 años, y la mediana fue 32 . La muestra estuvo compuesta por profe- 
sionales de la salud, particularmente psicólogos y estudiantes de psicología. El mayor número de participantes, 273, eran de nacionalidad argentina, 46 eran de Chile, 68 de Perú, 9 de Uruguay, 26 de Puerto Rico, 43 de México, 13 de Bolivia, 4 de Paraguay, 21 de Ecuador, 34 de Colombia, 28 de Venezuela y 61 del Caribe y Centroamérica. Con el propósito de satisfacer el modelo de promedios sujetos/variables (STV) basado en el número de variables que nuestro instrumento contiene, se determinó la necesidad de incluir por lo menos a 230 participantes en la muestra (Bryant \& Yarnold, 1995).

\section{Instrumentos}

El Imuder, en su forma preliminar, consta de 24 ítems y fue elaborado con el propósito de medir la presencia y frecuencia de ocurrencia de la depresión como un desorden de los componentes emocionales, cognitivos y conductuales de la personalidad y sus diferencias individuales (emociones positivas y emociones negativas) dentro del proceso del estrés percibido (Moscoso et al., 2003).

Los ítems del inventario están construidos de manera que reflejan la presencia de la depresión como una dimensión de características cognitivas y emociones negativas; y la ausencia de síntomas depresivos como expresión de un componente cognitivo y de emociones positivas. La escala estado evalúa la severidad del trastorno depresivo y la escala rasgo la frecuencia de ocurren- cia de este desorden. Las instrucciones son específicas para cada escala. En el caso de la escala de depresión/estado, se le solicita al participante indicar su respuesta en términos de cómo se siente en este momento, es decir, ;Ahora! y las opciones de respuesta son: 1. no en absoluto; 2. un poco; 3. bastante; 4. mucho. La escala de depresión/rasgo requiere que el participante marque su respuesta en términos de cómo se siente generalmente, es decir, la mayor parte del tiempo, y las posibilidades de respuesta son: 1. casi nunca; 2. algunas veces; 3. frecuentemente; 4. casi siempre.

El inventario de distrés emocional percibido (IDEP) consta de 15 ítems y fue elaborado con el propósito de medir la presencia y severidad del distrés emocional como un desorden cognitivo-afectivo. Este concepto es definido como "sentimientos subjetivos que varían en intensidad desde un estado de tristeza, incertidumbre, confusión y preocupación hasta la expresión de síntomas más severos como la ansiedad, depresión, ira, aislamiento social y desesperanza". El inventario mide tres dimensiones distintas del distres emocional: (1) ansiedad/ depresión, (2) desesperanza, y (3) la expresión de la ira. Dicho instrumento fue originalmente elaborado con el propósito de medir el distrés emocional percibido en pacientes con cáncer (Moscoso et al., 1999; Moscoso, McCreary, Goldenfarb, Knapp \& Reheiser, 2000; Moscoso \& Reheiser, 2010). Las respuestas a 
cada ítem del IDEP requiere que cada paciente reporte "en qué medida ha experimentado cada síntoma relacionado con el distrés emocional durante el último mes, incluyendo el día de hoy", indicando su respuesta en una escala de 3-puntos: (0) nunca; (1) a veces; (2) frecuentemente; (3) siempre.

El inventario de personalidad estadorasgo, forma revisada (STPI/R) de Spielberger (2010), es un cuestionario que contiene 80 ítems con el propósito de evaluar ansiedad, ira, curiosidad y depresión, tomando en consideración la distin ción estado y rasgo (Spielberger, Ritterband, Sydeman, Reheiser \& Unger, 1995). En nuestro estudio se utilizó únicamente la escala de depresión estado/ rasgo, el cual contiene 20 ítems derivados factorialmente en las subescalas de distimia y eutimia con el propósito de realizar los análisis de validez convergente.

El inventario de depresión de Beck, forma revisada $(\mathrm{BDI} / \mathrm{R})$, es una prueba psicométrica de 21 ítems elaborada con el propósito de evaluar el nivel de severidad de la depresión en sus componentes cognitivo, afectivo, conductual y somático (Beck \& Steer, 1987). La validez y confiabilidad de esta prueba ha sido establecida en pacientes con problemas psiquiátricos, así como también en poblaciones de adultos normales.

\section{Procedimiento}

La administración del conjunto de instrumentos se llevó a cabo en el Centro
Cultural General San Martín y en la Universidad Museo Social Argentino, sedes del congreso. La obtención de la muestra fue programada durante las "Sesiones de Poster" del evento, ofreciendo instrucciones verbales $\mathrm{y}$ escritas a todos los participantes. Se solicitó completar la página de información demográfica y responder a los 24 ítems del Imuder así como también los ítems de los cuestionarios y escalas que conformaron el paquete de evaluación. En cada caso la participación fue voluntaria y se mantuvo la anonimidad del participante. Cada persona completo el consentimiento de información.

\section{Resultados}

La validez factorial es un paso importante en el proceso de demostrar la consistencia interna del instrumento y observar adecuadamente las dimensiones de un orden mayor. Los ítems del Imuder fueron expuestos al análisis factorial exploratorio de componentes principales con rotaciones promax, tanto en mujeres como en varones. Decidimos utilizar el análisis factorial exploratorio en lugar del análisis factorial confirmatorio, debido a que el primero facilita un análisis más conservador (Gorsuch, 1988). Nuestro propósito fue observar si los componentes del instrumento, en ambas escalas, mantienen una adecuada estructura factorial y consistencia interna en una muestra poblacional no clínica de característi- 
cas multiculturales. Con la finalidad de facilitar la claridad de los datos empíricos, presentamos la estructura factorial no rotada de manera separada para las escalas de depresión/estado y depresión/rasgo. La muestra de nuestro estudio fue adecuada para proceder con el análisis factorial, como lo indican las medidas de adecuación muestral Kaiser-Meyer-Olkin $(\mathrm{KMO})>.60$. La medida de KMO fue aceptable con un valor de 0.93 , y la prueba de esfericidad de Bartlett fue significativa, indicando un valor $\mathrm{X}=7614.89(\mathrm{p}<0.001)$.

Como se puede observar en la tabla 1, el análisis de ejes principales para la Escala de Depresión/Estado nos muestra una solución factorial de un factor principal en ambos géneros, explicando un porcentaje de varianza del 33.16 para varones y 27.26 para mujeres. Así mismo, este factor principal presenta cargas factoriales superiores a $.43, \mathrm{y}$ un factor secundario con cargas factoriales menores de .29. Los doce ítems que componen la Escala de depresión/ Estado saturaron dentro del primer factor con cargas factoriales elevadas que varían entre .43 y 84 para varones (mediana $=.63$ ), así como, .59 y .88 para mujeres (mediana $=.74$ ). Es importante mencionar que los primeros seis ítems con contenido de afecto negativo, indicando la presencia de un estado depresivo, presentan cargas factoriales positivas, mientras que los otros seis ítems con contenido de afecto positivo tienen cargas factoriales negativas, demostran- do una correlación inversa. En el segundo factor, los doce ítems presentaron saturaciones insignificantes, por debajo de los criterios de retención de ítems. Las cargas factoriales en este segundo factor varían entre .21 y .29 para varones, así como .16 y .28 para mujeres. Es necesario indicar que en este análisis de ejes principales, únicamente el primer factor muestra valores eigen superiores a 1.00.

La tabla 2 presenta el análisis de ejes principales no rotados para la escala de depresión/rasgo. Al igual que para la escala depresión/estado, los análisis indican un solo factor tanto en varones como en mujeres, explicando un porcentaje de varianza de 39.16 para el sexo masculino, y del 24.16 para el sexo femenino. El factor I presenta también cargas factoriales superiores a .43. Todos los ítems que forman la escala de depresión/rasgo emergen como componentes de este primer factor con cargas factoriales definitivamente elevadas y que varían entre .43 y .88 para varones (mediana $=.65$ ). En el caso de las mujeres, dichas cargas factoriales fluctúan entre .49 y .82 (mediana $=.65$ ). Al igual que la escala de estado, debemos mencionar que los primeros seis ítems con contenido de afecto negativo, contienen cargas factoriales positivas, mientras que los otros seis ítems con contenido de afecto positivo, muestran cargas factoriales negativas confirmando, una vez más, la correlación inversa de estos dos grupos de ítems. El segundo factor está compuesto por los doce ítems de la 
escala, con saturaciones mínimas por debajo de los criterios de retención de ítems. Las cargas factoriales para este segundo factor fluctúan entre .18 y .32 para varones. En el caso de las mujeres, las cargas varían entre 16 y .32 .
Los resultados del análisis factorial de rotaciones promax de la escala de depresión- estado revelan una solución de dos factores claramente establecidos, tanto en varones como en mujeres. El primer factor está formado por seis

Tabla 1

Análisis factorial de ejes principales del inventario multicultural de la depresión, estado, para varones y mujeres $(\mathrm{N}=526)$

\begin{tabular}{|c|c|c|c|c|}
\hline \multirow{3}{*}{ *Ítems: Estado } & \multicolumn{4}{|c|}{ Ejes principales } \\
\hline & \multicolumn{2}{|c|}{ Factor 1} & \multicolumn{2}{|c|}{ Factor 2} \\
\hline & Varones & Mujeres & Varones & Mujeres \\
\hline 9- Siento deseos de quitarme la vida & .84 & .88 & .24 & .17 \\
\hline 11- Me siento deprimido (a) & .82 & .74 & .29 & .27 \\
\hline 1 - Me siento desilusionado (a) de la vida & .79 & .71 & .29 & .19 \\
\hline 3- He perdido las esperanzas de ser feliz & .71 & .76 & .27 & .19 \\
\hline 5- Me siento agobiado(a) por las dificultades & .66 & .74 & .23 & .24 \\
\hline $\begin{array}{l}\text { 7- No puedo dejar de pensar en mis proble- } \\
\text { mas }\end{array}$ & .43 & .64 & .22 & .16 \\
\hline 8- Me siento dichoso(a) & -.74 & -.76 & .27 & .18 \\
\hline 2- Me siento interesado en el futuro & -.62 & -.61 & .24 & .28 \\
\hline 12- Siento que disfruto la vida & -.76 & -.84 & .25 & .26 \\
\hline 6- Me siento contento(a) & -.85 & -.79 & .28 & .22 \\
\hline $\begin{array}{l}\text { 10- Me siento feliz en mis relaciones socia- } \\
\text { les }\end{array}$ & -.52 & -.70 & .21 & .20 \\
\hline $\begin{array}{l}\text { 4- Me siento entusiasmado con mis proyec- } \\
\text { tos }\end{array}$ & -.63 & -.59 & .29 & .17 \\
\hline Eigenvalues & 7.43 & 5.49 & .86 & .74 \\
\hline$\%$ de la varianza explicada & 33,16 & 27,26 & 1,18 & 1,26 \\
\hline
\end{tabular}

* Los ítems están ordenados de forma descendente según los pesos factoriales encontrados en la solución de ejes principales del factor 1 para varones.

* Los números asignados a los ítems corresponden al orden de los ítems en el inventario de evaluación (estado). 
ítems que describen un componente cognitivo/afectivo negativo, el cual conceptualizaremos como distrés. El segundo factor lo conforman también seis ítems que caracterizan una dimensión cognitiva/afectiva positiva. Este factor será denominado eustrés. Los resultados de estos análisis están reportados en la tabla 3 y representan la mejor y la más simple solución de dos factores. Por lo general se acepta que una solución factorial tiene una buena estructura simple cuando cada ítem, como en este caso, es asignado a un fac-

Tabla 2

Análisis factorial de ejes principales para los ítems del inventario multicultural de la depresión, rasgo, para varones y mujeres ( $\mathrm{N}=526$ )

\begin{tabular}{|c|c|c|c|c|}
\hline \multirow{3}{*}{ *Ítems: Rasgo } & \multicolumn{4}{|c|}{ Ejes principales } \\
\hline & \multicolumn{2}{|c|}{ Factor 1} & \multicolumn{2}{|c|}{ Factor 2} \\
\hline & Varones & Mujeres & Varones & Mujeres \\
\hline 5- Pienso que todo me va a salir mal & .88 & .82 & .21 & .30 \\
\hline 1- Soy una persona infeliz & .80 & .79 & .23 & .31 \\
\hline 3 - Siento que soy pesimista & .74 & .49 & .19 & .27 \\
\hline 9- Me siento culpable si no logro mis metas & .74 & .70 & .24 & .16 \\
\hline 11- Siento que soy apático(a) & .69 & .74 & .18 & .22 \\
\hline 7- Le doy cien vueltas a mis problemas & .43 & .66 & .26 & .32 \\
\hline 4- Soy una persona llena de vitalidad & -.66 & -.72 & .28 & .30 \\
\hline 12- Soy una persona alegre & -.86 & -.81 & .30 & .31 \\
\hline 10- Pienso que mi futuro es brillante & -.74 & -.78 & .29 & .22 \\
\hline 6- Siento que soy una persona sociable & -.62 & -.56 & .32 & .24 \\
\hline 2- Me encantan mis actividades laborales & -.51 & -.54 & .18 & .16 \\
\hline 8- Pienso que los fracasos son parte de la vida & -.63 & -.69 & .29 & .29 \\
\hline Eigenvalues & 8.42 & 7.76 & .94 & .67 \\
\hline$\%$ de la varianza explicada & 39,16 & 24,16 & 1,56 & 1,08 \\
\hline
\end{tabular}

* Los ítems están ordenados de forma descendente según los pesos factoriales encontrados en la solución de ejes principales del factor 1 para varones.

* Los números asignados a los ítems corresponden al orden de los ítems en el inventario de evaluación (rasgo). 
tor único sin ningún tipo de ambigüedad o saturaciones dobles (Nunnally, 1978). Los análisis de rotaciones promax nos muestran que ambos factores en esta escala presentan valores eigen superiores a 1.00 .
Las cargas factoriales del factor I (distrés) son bastante elevadas, variando entre .56 y .88 para varones (mediana $=$ .72), y .61 a .89 para mujeres (mediana= .75). El coeficiente alpha de Cronbach de este primer factor es de .89 para

Tabla 3

Análisis factorial de rotaciones promax, coeficientes alpha de Cronbach y correlaciones de ítems-puntuación total (IRs) del inventario multicultural de la depresión, estado, para varones y mujeres ( $N=526$ )

\begin{tabular}{|c|c|c|c|c|c|c|}
\hline \multirow{3}{*}{ Ítems*: Estado } & \multicolumn{4}{|c|}{ Rotaciones promax } & & \\
\hline & \multicolumn{2}{|c|}{ Factor $1 /$ distrés } & \multicolumn{2}{|c|}{ Factor 2/eustrés } & \multicolumn{2}{|c|}{$\begin{array}{l}\text { Correlaciones } \\
\text { ítem-total (IRs) }\end{array}$} \\
\hline & Varones & Mujeres & Varones & Mujeres & Varones & Mujeres \\
\hline $\begin{array}{l}\text { 9- Siento deseos de quitar- } \\
\text { me la vida }\end{array}$ & .76 & .80 & & & .70 & .72 \\
\hline 11- Me siento deprimido (a) & .86 & .89 & & & .89 & .83 \\
\hline $\begin{array}{l}1 \text { - Me siento desilusionado } \\
\text { (a) de la vida }\end{array}$ & .72 & .67 & & & .71 & .69 \\
\hline $\begin{array}{l}\text { 3- He perdido las esperan- } \\
\text { zas de ser feliz }\end{array}$ & .88 & .83 & & & .86 & .81 \\
\hline $\begin{array}{l}\text { 5- Me siento agobiado(a) } \\
\text { por las dificultades }\end{array}$ & .56 & .61 & & & .65 & .67 \\
\hline $\begin{array}{l}\text { 7- No puedo dejar de pen- } \\
\text { sar en mis problemas }\end{array}$ & .71 & .69 & & & .71 & .70 \\
\hline 8- Me siento dichoso(a) & & & .76 & .81 & .69 & .78 \\
\hline $\begin{array}{l}\text { 2- Me siento interesado(a) } \\
\text { en el futuro }\end{array}$ & & & .79 & .84 & .76 & .81 \\
\hline $\begin{array}{l}\text { 12- Siento que disfruto la } \\
\text { vida }\end{array}$ & & & .67 & .75 & .66 & .71 \\
\hline 6- Me siento contento(a) & & & .61 & .64 & .65 & .67 \\
\hline $\begin{array}{l}\text { 10- Me siento feliz en mis } \\
\text { relaciones sociales }\end{array}$ & & & .68 & .74 & .65 & .69 \\
\hline $\begin{array}{l}\text { 4- Me siento entusiasmado } \\
\text { con mis proyectos }\end{array}$ & & & .70 & .66 & .68 & .67 \\
\hline Eigenvalues & 4.23 & 3.16 & 1.98 & 1.21 & & \\
\hline $\begin{array}{l}\text { Coeficientes Alpha } \\
\text { (Cronbach) }\end{array}$ & 89 & .86 & .87 & .84 & & \\
\hline
\end{tabular}

* Los números asignados a los ítems corresponden al orden de los ítems en el inventario de evaluación (Estado). 
varones y .86 para mujeres, lo que nos indica claramente un elevado nivel de consistencia interna de los ítems. El factor II de esta escala (eustrés) presenta cargas factoriales que oscilan entre .61 y .79 para varones (mediana $=.75$ ), y de .64 a .84 para mujeres (mediana= .74). El coeficiente de Cronbach's alpha para el segundo factor indica .87 para varones y .84 para mujeres, demostrando empíricamente que los ítems son de naturaleza homogénea. Es importante indicar que no existieron saturaciones dobles con pesos superiores a 0.30. Las correlaciones item-puntuación total (IR's) de los reactivos que representan la escala estado son esencialmente elevadas, y en su mayoría con valores mayores a .70. Estas correlaciones oscilan entre .65 y .89 para varones, así como .67 y .83 para mujeres.

La tabla 4 nos muestra la estructura factorial de la escala de depresiónrasgo en base al análisis factorial de rotaciones promax. Estos datos indican una solución de dos factores con valores eigen superiores a 1.00, tanto en varones, como en mujeres. El factor I de esta escala la componen seis ítems que representan aspectos cognitivos y afectivos de carácter negativo, los cuales en su conjunto indican una predisposición de personalidad vulnerable a experimentar estados de distrés emocional y trastornos depresivos. En base a nuestro modelo conceptual, este factor es denominado distrés. El factor II lo conforman, de igual manera, seis ítems que caracterizan una dimensión cognitiva/ afectiva positiva y que describen una predisposición de personalidad adaptable, resiliente, y con un buen grado de efectividad de afrontamiento. Estas características conductuales representan un estado de bienestar, por lo tanto, este factor es reconocido como eustrés.

Las cargas factoriales del primer factor reconocido como distrés son significativamente elevadas oscilando entre .56 y .81 para varones (mediana= .68), y .69 a .87 en mujeres (mediana= .78). El coeficiente alpha de Cronbach de este primer factor es de .86 para varones y .89 para mujeres, indicando un elevado nivel de consistencia interna de los ítems. El segundo factor de esta escala denominado eustrés presenta cargas factoriales que oscilan entre $.52 \mathrm{y}$ .82 para varones (mediana $=.67$ ), y de .59 a .83 para mujeres (mediana $=.71$ ). El coeficiente de Cronbach's alpha para este segundo factor es de .91 para varones y .84 para mujeres, lo cual demuestra que los ítems son de naturaleza homogénea. Al igual que en la escala estado, es interesante observar que en esta escala/rasgo no se presentaron saturaciones dobles con pesos superiores a 0.30 como criterio de corte para los datos reportados en el presente análisis. Las correlaciones ítem-puntuación total (IR) de los ítems que representan la escala rasgo son altas con valores mayores de .60. Estas correlaciones oscilan entre .61 y .82 para varones, así como .61 y .85 para las mujeres. 
Tabla 4

Análisis factorial de rotaciones promax, coeficientes alpha de Cronbach y correlaciones de ítems-puntuación total (IRs) del inventario multicultural de la depresión, rasgo, para varones y mujeres $(\mathrm{N}=526)$

\begin{tabular}{|c|c|c|c|c|c|c|}
\hline \multirow{3}{*}{ *Ítems: Rasgo } & \multicolumn{4}{|c|}{ Rotaciones Promax } & \multirow{2}{*}{\multicolumn{2}{|c|}{$\begin{array}{l}\text { Correlaciones } \\
\text { ítem-total (IRs) }\end{array}$}} \\
\hline & \multicolumn{2}{|c|}{ Factor 1/Distres } & \multicolumn{2}{|c|}{ Factor 2/Eustres } & & \\
\hline & Varones & Mujeres & Varones & Mujeres & Varones & Mujeres \\
\hline $\begin{array}{l}\text { 5- Pienso que todo me va } \\
\text { a salir mal }\end{array}$ & .81 & .84 & & & .79 & .79 \\
\hline 1- Soy una persona infeliz & .80 & .83 & & & .78 & .81 \\
\hline $\begin{array}{l}3 \text { - Siento que soy pesimis- } \\
\text { ta }\end{array}$ & .76 & .82 & & & .74 & .79 \\
\hline $\begin{array}{l}\text { 9- Me siento culpable si no } \\
\text { logro mis metas }\end{array}$ & .78 & .87 & & & .73 & .81 \\
\hline $\begin{array}{l}\text { 11- Siento que soy apático } \\
\text { (a) }\end{array}$ & .66 & .69 & & & .64 & .67 \\
\hline $\begin{array}{l}\text { 7- "Le doy cien vueltas" a } \\
\text { mis problemas }\end{array}$ & .56 & .78 & & & .61 & .76 \\
\hline $\begin{array}{l}\text { 4- Soy una persona llena } \\
\text { de vitalidad }\end{array}$ & & & .52 & .61 & .61 & .62 \\
\hline 12- Soy una persona alegre & & & .82 & .78 & .76 & .71 \\
\hline $\begin{array}{l}\text { 10- Pienso que mi futuro es } \\
\text { brillante }\end{array}$ & & & .64 & .59 & 62 & .61 \\
\hline $\begin{array}{l}\text { 6- Siento que soy una per- } \\
\text { sona sociable }\end{array}$ & & & .58 & .72 & .61 & .71 \\
\hline $\begin{array}{l}\text { 2- Me encantan mis activi- } \\
\text { dades laborales }\end{array}$ & & & .76 & .83 & .71 & .72 \\
\hline $\begin{array}{l}8 \text { - Pienso que los fracasos } \\
\text { son parte de la vida }\end{array}$ & & & .81 & .75 & .77 & .69 \\
\hline Eigenvalues & 5.65 & 6.12 & 2.54 & 3.67 & & \\
\hline $\begin{array}{l}\text { Coeficientes Alpha } \\
\text { (Cronbach) }\end{array}$ & .86 & .89 & .91 & .84 & & \\
\hline
\end{tabular}

* Los números asignados a los ítems corresponden al orden de los ítems en el inventario de evaluación.

\section{DISCUSIÓN Y CONCLUSIONES}

El propósito principal de este estudio fue presentar el modelo conceptual que sirvió de guía en la construcción y adaptación de los ítems del inventario multicultural de la depresión, estadorasgo (Imuder), en su forma preliminar, así como también reportar la estructura 
factorial, validez de constructo, y consistencia interna de las dos escalas del instrumento en una muestra poblacional no clínica y multicultural de adultos de habla hispana. Se decidió utilizar el análisis factorial exploratorio, en lugar de análisis factorial confirmatorio, debido a que el primero facilita un tipo de análisis más conservativo (Gorsuch, 1988).

La construcción del Imuder se sustenta en el modelo conceptual de Susan Folkman (1997) acerca de la coexistencia de emociones positivas y emociones negativas dentro del proceso del estrés severo y la depresión. El marco conceptual de Folkman se vio significativamente reforzado por los estudios de Zautra (2003) y el soporte empírico ofrecido por los estudios de Fredickson (1998), Seligman \& Csikszentmihalyi (2000) y Watson \& Clark (1994).

Los resultados del análisis factorial de componentes principales, sin rotación, realizados en forma separada para la escala estado como para la escala rasgo, nos indican que el Imuder es un instrumento psicométrico que presenta una sólida y robusta validez de constructo. Estos datos sugieren que el Imuder provee información significativa acerca de las diferencias individuales en términos de emociones positivas y emociones negativas que moderan, o en su defecto predisponen a experimentar un estado depresivo bajo condiciones de estrés.

El análisis factorial de componentes principales con rotaciones promax de los 24 ítems del Imuder indica la existencia de dos factores no correlacionados en la escala estado, y de dos factores, igualmente no correlacionados en la escala rasgo. La aparición de estos dos factores, a los cuales denominamos distrés y eustrés representan la mejor, la más simple, y la más significativa información de las dimensiones cognitiva y emocional de la depresión en el contexto del estrés percibido. La denominación de distrés en referencia a los doce ítems que contienen una connotación negativa o sugestiva de la presencia de un trastorno depresivo, es consistente con los conceptos y resultados de las investigaciones realizadas por Folkman y colaboradores, así como también del modelo tripartito y el factor de distrés general presentado por Clark \& Watson (1991).

La estructura factorial del instrumento confirma psicométricamente las propiedades estructurales asumidas en nuestros planteamientos hipotéticos y ofrece soporte empírico a la conceptualización de la depresión como un constructo mediado por la interacción de rasgos cognitivos y emocionales de la personalidad. Los 24 ítems del Imuder presentan cargas factoriales significativamente elevadas, y toman en consideración el significado de la depresión como un concepto coherente en respuesta a la coexistencia de las emociones positivas y negativas dentro del proceso de estrés percibido. Es importante resaltar que la selección 
de estos 24 ítems expuestos al análisis factorial exploratorio, responde a la brillante y efectiva labor de los jueces durante el proceso de evaluación de la validez de contenido en nuestro estudio piloto. En este sentido, decidimos retener los 24 ítems expuestos al análisis factorial exploratorio con el propósito de continuar evaluando sus propiedades psicométricas en futuros estudios transculturales.

A pesar del impresionante nivel de validez de constructo y consistencia interna reportada en este estudio, nuestros resultados deben ser entendidos como un reporte preliminar y con las limitaciones que representan los estudios iniciales. Sin lugar a dudas, estos resultados nos estimulan a continuar examinando las propiedades psicométricas del Imuder y seguir esforzándonos con el propósito de replicar estos mismos resultados con muestras más amplias y diversas en países de América Latina.

\section{ReferenCiAs}

American Psychiatric Association (2000). Diagnostic and statistical manual of mental disorders. ( $4^{\mathrm{a}}$ ed.). Washington, DC: American Psychological Association.

Beck, A. T., et al. (2011). Beyond depression: Toward a process-based approach to research, diagnosis and treatment. Clinical Psychology Science and Practice, 18, 275-299.
Beck, A. T., \& Steer, R. (1987). Beck depression inventory: Manual. Nueva York, NY: Psychological Corp.

Beck, A. T., \& Steer, R. (1993). Manual for Beck depression inventory. San Antonio, TX: Psychological Corp.

Beck, A. T., Steer, R., \& Brown, G. K. (1996). Beck depression inventory: Manual BDI-II. Nueva York, NY: Psychological Corp.

Bolger, N., \& Zuckerman, A. (1995). A framework for studying personality in the stress process. Journal of Personality and Social Psychology, 69, 890-902.

Bryant, B. F., \& Yarnold, P. R. (1995). Principal components analysis and exploratory and confirmatory factor analysis. En L. G. Grimm \& P. R. Yarnold (Eds.), Reading and understanding multivariate statistics (pp. 99-136). Washington, DC: American Psychological Association.

Cattell, R.B., \& Sheier, I.H. (1961). The Meaning and Measurement of Neuroticism and Anxiety. New York, NY: Ronald Press.

Clark, L.A., \& Watson, D. (1991). Tripartite model of anxiety and depression: Psychometric evidence and taxonomic implications. Journal of Abnormal Psychology, 100, 316-336.

Diener, E., Sandvic, E., \& Pavot, W. (1991). Happiness is the frequency, not intensity, of positive vs. negative affect. En F. Strack, M. Argyle \& N. 
Schwarz (Eds.), The Social Psychology of Subjective Wellbeing (pp. 119-139). Nueva York, NY: Pergamon.

Fava, M. (2003). Diagnosis and definition of treatment-resistant depression. Biological Psychiatry, 53, 649659.

Folkman, S. (1997). Positive psychological states and coping with severe stress. Social Science Medicine, 45(8), 1207-1221.

Folkman, S. (2008). The case for positive emotions in the stress process. Anxiety, Stress and Coping, 21(1), 3-14.

Fredrickson, B. L. (1998). What good are positive emotions? Review of General Psychology, 2, 300-319.

Fredrickson, B. L. (2003). The value of positive emotions. American Scientist, 91, 330-335.

Gorsuch, R. L. (1988). Exploratory factor analysis. En J. R. Nesselroade \& R. B. Cattell (Eds.), Handbook of multivariate experimental psycho$\operatorname{logy}$ ( $2^{\text {a }}$ ed.), pp. 231-258). Nueva York, NY: Plenum Press.

Insel, T., et al. (2010). Research domain criteria: Toward a new classification framework for research on mental disorders. American Journal of Psychiatry, 167, 748-751.

Kendall, P. C., \& Watson, D. (1989). Anxiety and depression: Distinctive and overlapping features. San Diego, CA: Academic Press.

Kendler, K., \& Prescott, C. (1998). Stressful life events and major depression: Risk period, long-term contextual threat and diagnostic specificity. Journal of Nervous and Mental Disorders, 186, 661-669.

Kessler, R. C., et al. (2005). Lifetime prevalence and age of onset distributions of DSM-IV disorders in the national comorbity survey replication. Archives of General Psychiatry, 62, 593-602.

Larson, C. L., Nitschke, J. B., \& Davidson, R. J. (2007). Common and distinct patterns of effective responses in dimensions of anxiety and depression. Emotion, 7, 182-191.

Lazarus, R. S., \& Folkman, S. (1984). Stress, appraisal, and coping. Nueva York, NY: Springer Publishing.

Mallon, L., \& Hetta, J. (2002). Detección de la depresión mediante cuestionarios: Comparación de una sola pregunta con los datos de la entrevista en una muestra de adultos de edad avanzada. European Journal of Psychiatry, 16, 139-148.

Mayberg, H. S. (2004). Depression: A neuropsychiatric perspective. En J. Panksepp (Ed.), Textbook of biological psychiatry (pp. 197-229). Hoboken, NJ: Wiley.

Mazure, C. H. (1998). Life stressors as risk factors in depression. Clinical 
Psychology and Science Practice, 5, 291-313.

Moscoso, M. S. (2000). Estructura factorial del inventario multicultural latinoamericano de la expresión de la cólera y hostilidad. Revista Latinoamericana de Psicología, 32(2), 321-343.

Moscoso, M. S., Knapp, M., \& Llauget, J. (2003). The preliminary manual State-Trait Multicultural Depression Inventory: A guide to its development and use. Tampa, FL: University of South Florida. Unpublished publication manual. Available from the authors.

Moscoso, M. S., McCreary, D., Goldenfarb, P., Knapp, M., \& Reheiser, E. C. (2000). A brief screening inventory to measure emotional distress in cancer patients. Psycho-Oncolo$g y$, , S4.

Moscoso, M. S., McCreary, D., Goldenfarb, P., Knapp, M., \& Rohr, J. (1999). Construction of an inventory to measure emotional distress in cancer patients. Psycho-Oncology, 8 , S53.

Moscoso, M. S., \& Reheiser, E. C. (2010). Construct validity of the emotional distress inventory in patients with cancer. Ansiedad y Estrés, 16(1), 83-94.

Moscoso, M. S., \& Spielberger, C. D. (1999). Measuring the experience, expression and control of anger in
Latin America: The Spanish multicultural State-Trait Anger Expression Inventory. Interamerican Journal of Psychology, 33, 29-48.

Nunnally, J. C. (1978). Psychometric theory (2a ed.). Nueva York, NY: McGraw-Hill.

Ritterband, L. M., \& Spielberger, C. D. (1996). Construct validity of the Beck depression inventory as a measure of stait and trait depression in nonclinical populations. Depression and Stress, 2, 123-145.

Rosenfeld, I. (1999). When the sadness won't go away. Parade Magazine, 19, 10.

Seligman, M. E., \& Csikszentmihalyi, M. (2000). Positive Psychology. An introduction. American Psychologist, 55, 5-14.

Spielberger, C. D. (1988). Manual for the state-trait anger expression inventory (STAXI). Odessa, FL: Psychological Assessment Resources.

Spielberger, C. D., et al. (1979). The preliminary manual for the state-trait personality inventory. Tampa, FL: University of South Florida, Unpublished publication manual.

Spielberger, C. D., Carretero-Dios, H., De los Santos-Roig, M., \& BuelaCasal, G. (2002). Spanish experimental version of the Stait-Trait Depression Questionnaire: State Subscale. International Journal of Health and Psychology, 2, 7-34. 
Spielberger, C. D., Gorsuch, R., \& Luschene, R. (1970). Manual for the State-Trait Anxiety Inventory. Palo Alto, CA: Consulting Psychologists Press.

Spielberger, C. D., Moscoso, M. S., \& Brunner, T. M. (2005). Cross-cultural assessment of emotional states and personality traits. En R. K. Hambleton, P. F. Merenda, \& C.D. Spielberger (Eds.), Adapting Educational and Psychological Tests for Cross-Cultural Assessment (pp 343-367). Mahwah, NJ: Lawrence Erlbaum.

Spielberger C. D., \& Reheiser E. C. (2010). Assessment of emotions: Anxiety, anger, depression, and curiosity. Applied Psychology: Health and Wellness, 1, 32.

Spielberger, C. D., Reheiser, E. C., Owen, A. E., \& Sydeman, S. J. (2003). Measuring psychological vital signs of anxiety, anger, depression, and curiosity in treatment planning and outcome assessment. En M. Marquis (Ed.), The use of psychological testing for treatment, planning and outcomes assessment ( $3^{\mathrm{a}}$ ed.), (pp. 421-447). Hillsdale, NJ: Lawrence Erlbaum Assoc.

Spielberger, C. D., Ritterband, L. M., Reheiser, E. C., \& Brunner, T. M. (2003). The nature and measurement of depression. International Journal of Clinical and Health Psychology, 3, 209-234.

Spielberger, C. D., Ritterband, L. M., Sydeman, S. J., Reheiser, E. C., \& Unger, K. K. (1995). Assessment of emotional states and personality traits: Measuring psychological vital signs. En J.N. Butcher (Ed.) Clinical Personality Assessment: Practical Approaches (pp. 42-58). Nueva York, NY: Oxford University Press.

Watson, D. \& Clark, L. A. (1994). Manual for the positive and negative affect schedule (expanded form). Iowa, IA: University of Iowa; available from the authors.

World Health Organization. (2008). Global Burden of Disease: 2004 Update.

Zautra, A. J. (2003). Emotions, stress and health. Nueva York, NY: Oxford University Press.

Zautra., A. J., \& Smith, B. W. (2001). Depression and reactivity to stress in older women with rheumatoid arthritis and osteoarthritis. Psychosomomatic Medicine, 63, 687-696.

Zautra, A. J., Affleck, G. G., Tennen, H., Reich, J. W., \& Davis, M. C. (2005). Dynamic approaches to emotions and stress in every day life: Bolger and Zuckerman reloaded with positive as well as negative affects. Journal of Personality, 73(6), 1511-1538. 